\title{
Acute Inferior Wall ST Segment Elevation Myocardial Infarction Caused Atrial Lead Dysfunction Reversed by Primary Percutaneous Coronary Intervention
} A Case Report

\author{
Hui-Ting Wang, ${ }^{1,3} \mathrm{MD}$, Yung-Lung Chen, ${ }^{2,3} \mathrm{MD}$ and Chiung-Jen $\mathrm{Wu},{ }^{2,3} \mathrm{MD}$
}

\begin{abstract}
Summary
The mechanism of atrial lead dysfunction varies in patients receiving pacemaker implantation and this needs to be investigated, especially when the causes are reversible. We report and discuss a 76-year-old female who had atrial lead dysfunction caused by acute myocardial infarction and who was recovered after primary percutaneous coronary intervention. The sequential electrocardiographic changes were demonstrated and the possible mechanisms were discussed.
\end{abstract}

(Int Heart J 2018; 59: 618-621)

Key words: Acute myocardial infarction, Sick sinus syndrome, Atrioventricular block, Pacemaker

$\mathrm{T}$ he number of pacemaker implantations is increasing in the world as the general population ages. ${ }^{1}$ Many of these patients with permanent pacemakers may suffer from myocardial ischemia or infarction. Myocardial ischemia or infarction is one of the possible causes for lead dysfunction, which may be underestimated and could be reversible. We report and discuss the case of a 76-year-old female who had atrial lead dysfunction caused by acute myocardial infarction and who was recovered after primary percutaneous coronary intervention. The sequential electrocardiographic changes are demonstrated and the possible mechanisms are discussed.

\section{Case Report}

A 76-year-old Asian female was sent to the emergency department of a medical center due to general weakness and chest tightness. After reviewing her medical chart it was discovered that she had history of end-stage renal disease (under maintenance of hemodialysis), triplevessel coronary artery disease (with percutaneous coronary angioplasty), urothelial carcinoma (with nephroureterectomy), type 2 diabetes mellitus, and hypertension. Two months previously, she received dual chamber pacemaker implantation (Boston Scientific, ALTRUA REF: S 603DR) due to sick sinus syndrome confirmed by electrophysiologic study (EPS) with sinus node recovery time $4779 \mathrm{~ms}$ at rapid high right atrial pacing with cycle length of $300 \mathrm{~ms}$. Prolonged AV node conduction (AH:167 ms; HV: $61 \mathrm{~ms}$ ) was also confirmed by EPS. The atrial lead was Metronic $(5554-53 \mathrm{~cm})$ passive-fixation bipolar elec- trode, and the ventricular lead was St. Jude Medical (Tendril $1888 \mathrm{TC} / 58 \mathrm{~cm}$ ) active-fixation bipolar electrode. The original pacemaker setting was DDDR mode with dynamic AV delay at minimal delay $150 \mathrm{~ms}$ and maximal delay $300 \mathrm{~ms}$. The magnet electrocardiography (ECG) (DOO mode) on the second day of pacemaker implantation is shown in Figure 1A, which shows AV sequential pacing with rate of $100 \mathrm{BPM}$ and pacing AV interval 120 ms. The ST segment was flat at that time.

On arrival, her blood pressure obtained at triage was $63 / 49 \mathrm{mmHg}$ and her heart rate was 60 beats/minute. The ECG upon arrival showed ventricular pacing with ST segment elevation over lead II, III and aVF (compared with Figure 1A) and pacemaker dysfunction with atrial undersensing and pacing spike without capture (Figure 1B). She was categorized as Killip class IV due to hypotension. Primary percutaneous coronary intervention (PCI) was performed for the inferior wall ST segment elevation myocardial infarction (STEMI) immediately, which showed that proximal right coronary artery (RCA) previous drug-eluting stent had in-stent significant restenosis with thrombus burden (Figure 2A). Balloon angioplasty with a smaller $2 \times 20 \mathrm{~mm}$ MINI TREK balloon inflation over proximal RCA in-stent restenosis site from 16 to 20 atms gradually, and then a bigger $2.5 \times 20 \mathrm{~mm}$ Hiryu balloon inflation from 28 to 30 atms, was performed smoothly without complication. After balloon angioplasty, the right atrial branch of RCA showed significantly improved blood flow (Figure 2B). Intermittent atrial undersensing and atrial pacing spike with regained capture but pronounced latency were noticed by serial ECG just

From the 'Department of Emergency, Kaohsiung Chang Gung Memorial Hospital, Kaohsiung, Taiwan, ${ }^{2}$ Division of Cardiology, Department of Internal Medicine, Kaohsiung Chang Gung Memorial Hospital, Kaohsiung, Taiwan and ${ }^{3}$ Chang Gung University College of Medicine, Kaohsiung, Taiwan.

Address for correspondence: Yung-Lung Chen, MD or Chiung-Jen Wu, MD, Division of Cardiology, Department of Internal Medicine, Kaohsiung Chang Gung Memorial Hospital, 123 Ta Pei Road, Niao Sung Hsiang, Kaohsiung 83301, Taiwan. E-mail: feymanchen@yahoo.com.tw or lung@adm.cgmh.org.tw

Received for publication May 16, 2017. Revised and accepted August 3, 2017.

Released in advance online on J-STAGE April 6, 2018.

doi: $10.1536 /$ ihj. $17-270$

All rights reserved by the International Heart Journal Association. 


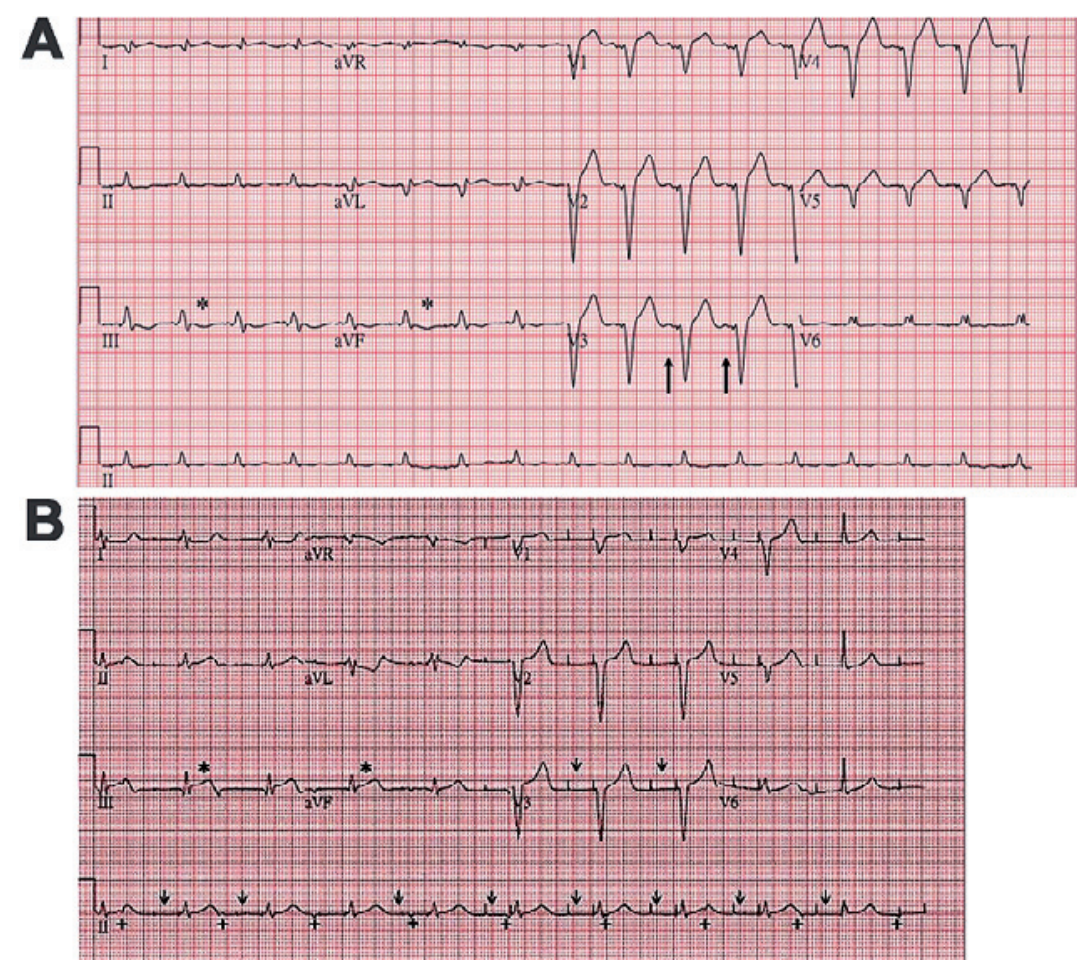

Figure 1. A: Electrocardiogram tracing on the second day of pacemaker implantation reveals atrioventricular sequential pacing with good magnet response (DOO mode with pacing rate of $100 \mathrm{bpm}$ ). The arrow indicates normal atrial capture. The star symbol indicates flat ST segment. B: The ECG obtained upon ED arrival showed ventricular pacing with ST segment elevation over lead II, III, and aVF (compared with A). The star symbols indicate new ST segment elevation. The arrow indicates pacing spike without capture. The plus symbols indicate low atrial rhythm with under-sensing.

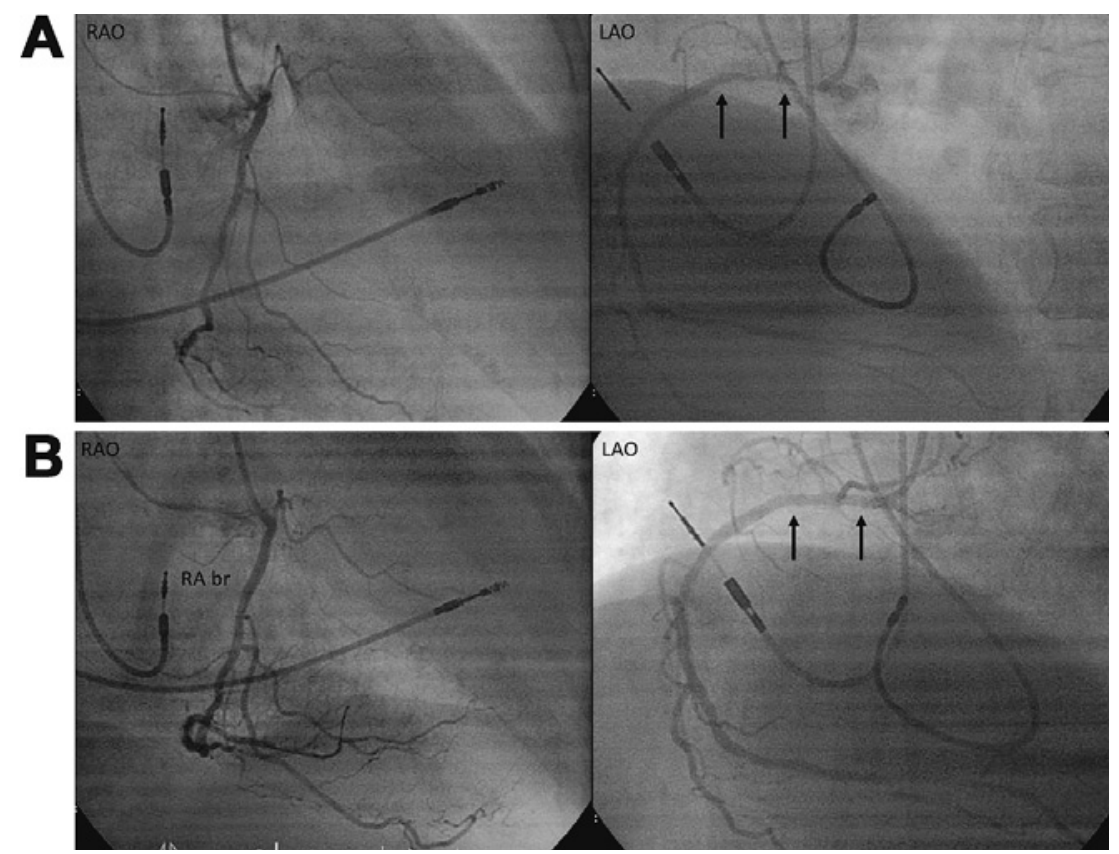

Figure 2. A: The ostium to proximal of right coronary artery (RCA) revealed in-stent restenosis. There is also distal RCA total occlusion at in-stent site. The arrows indicate critical lesion over RCA. B: The fluoroscopy taken after primary percutaneous coronary intervention (PCI). The arrows indicate restoration of patency over the previous critical lesions over RCA. RA br. represents right atrial branch of RCA, which revealed significantly improved blood flow after the PCI. 


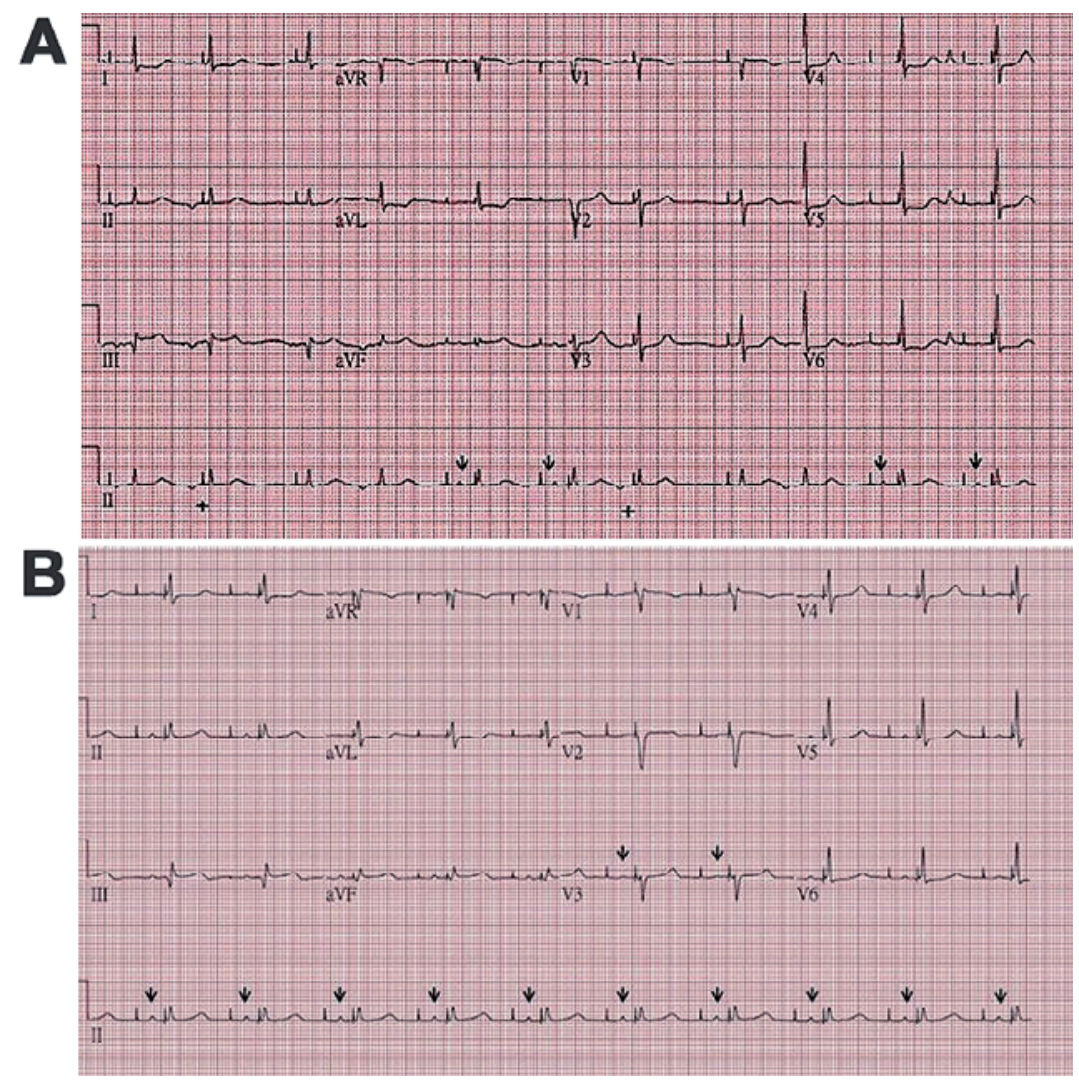

Figure 3. A: The electrocardiogram obtained after primary stenting that reveals intermittent atrial undersensing and atrial pacing spike with capture but latency. The plus symbols reveal undersensing of atrial contraction. The arrows indicate atrial pacing spike with capture but pronounced latency. B: Follow-up electrocardiogram shows normal atrial pacing function with only pronounced latency.

after primary stenting (Figure 3A). Pacemaker follow-up with programmer at the 2 nd day of primary PCI showed that sensing and pacing parameters of leads were within normal range. The follow-up ECG showed normal atrial pacing function with only pronounced latency on the second day after PCI (Figure 3B).

\section{Discussion}

Dual chamber pacemakers are indicated in patients with sick sinus syndrome (SSS) and advanced AV block. ${ }^{1}$ The incidence of pacemaker dysfunction ranges from $0.46 \%$ to $2 \%{ }^{2,3)}$ Pacing system malfunction can be due to malfunction of lead, electrode-tissue interface, or pulse generator. The mechanism of leads dysfunction varies and includes leads issue, the condition of patients and electromagnetic interference (EMI). ${ }^{4}$

The implantation of a new lead is indicated only after the reversible etiology is excluded. Besides, the diagnosis of STEMI is also challenging in patients with permanent pacemakers, and this may cause delayed therapy. ${ }^{5,6)}$ We have reported this case to increase the awareness that ischemia or infarction is one of the possibilities leading to cause of lead dysfunction which may be underestimated, and could be reversed after revascularization.
According to the literature review, there are two cases with regard to atrial lead dysfunction and myocardial ischemia. Chen, et al. described a case of non-ST segment elevation myocardial infarction following by atrial lead dysfunction.7) They proposed a hypothesis that the transient atrial electrophysiological dysfunction may result from the change of the threshold of electrode-tissue interface in the right atrium. Upadhyay, et al. reported a case with inferior wall STEMI causing transient loss of sensing and capture of the atrial lead of a permanent dualchamber pacemaker, and hypothesized that ischemia of the right atrium caused stunning of the atrial myocardium at the pacer-lead interface. ${ }^{8)}$ Both cases had persistent atrial lead dysfunction after revascularization and the resumption of the lead function was delayed for more than 40 days. To the best of our knowledge, this is the first case with instant improvement of atrial lead dysfunction after primary PCI. Acute myocardial infarction may induce pacing lead dysfunction by causing atrial ischemia. Timely primary coronary intervention especially in case of STEMI is the treatment of choice to reverse the pacing or sensing failure. 


\section{Disclosures}

Conflicts of interest: None.

\section{References}

1. Epstein AE, DiMarco JP, Ellenbogen KA, et al. ACC/AHA/HRS 2008 Guidelines for Device-Based Therapy of Cardiac Rhythm Abnormalities: a report of the American College of Cardiology/ American Heart Association Task Force on Practice Guidelines (Writing Committee to Revise the ACC/AHA/NASPE 2002 Guideline Update for Implantation of Cardiac Pacemakers and Antiarrhythmia Devices): developed in collaboration with the American Association for Thoracic Surgery and Society of Thoracic Surgeons. Circulation 2008; 117: e350-408.

2. Maisel WH, Moynahan M, Zuckerman BD, et al. Pacemaker and ICD generator malfunctions: analysis of Food and Drug Administration annual reports. JAMA 2006; 295: 1901-6.

3. Hauser RG, Hayes DL, Kallinen LM, et al. Clinical experience with pacemaker pulse generators and transvenous leads: an 8 year prospective multicenter study. Heart rhythm 2007; 4: 15460.

4. Nakai T, Kurokawa S, Ikeya Y, et al. MRI Mode Programming for Safe Magnetic Resonance Imaging in Patients With a Magnetic Resonance Conditional Cardiac Device. Int Heart J 2016; 57: $173-6$

5. Bertel N, Witassek F, Puhan M, et al. Management and outcome of patients with acute myocardial infarction presenting with pacemaker rhythm. Int J Cardiol 2017; 230: 604-9.

6. Lee PT, Chao TH, Huang YL, et al. Analysis of the Clinical Characteristics, Management, and Causes of Death in Patients with ST-Segment Elevation Myocardial Infarction Treated With Primary Percutaneous Coronary Intervention from 2005 to 2014. Int Heart J 2016; 57: 541-6.

7. Chen C, Fang CC, Wang SP. Reversible atrial lead dysfunction of DDD pacemaker after recent inferior myocardial infarctiona case report. Angiology 2004; 55: 451-4.

8. Upadhyay S, Marshalko S, McPherson C. The stunned atrial lead: Transient malfunction of a permanent atrial pacer lead following acute myocardial infarction. Int J Crit Illn Inj Sci 2011; 1: 161-3. 